\title{
A NECESSARY AND SUFFICIENT CONDITION FOR $w^{*}$-BOUNDED SETS TO BE STRONGLY BOUNDED
}

\author{
CARLOS BOSCH AND JAN KUCERA \\ (Communicated by William J. Davis)
}

\begin{abstract}
It is known that if a locally convex space $E$ is quasi-complete then every $\sigma\left(E^{\prime}, E\right)$-bounded set in $E^{\prime}$ is $\beta\left(E^{\prime}, E\right)$-bounded. This result cannot be reversed. Here we show that every $\sigma\left(E^{\prime}, E\right)$-bounded set is $\beta\left(E^{\prime}, E\right)$-bounded iff $E$ is fast complete.
\end{abstract}

Throughout the paper $E$ is a locally convex space. In accordance with [1] we call a balanced convex set $B \subset E$ a disk. For a disk $B \subset E$ we denote by $E_{B}$ the linear hull of $B$ and provide it with the topology generated by the basis $\{(1 / n) B ; n \in N\}$. If the normed space $E_{B}$ is Banach, we call $B$ a Banach disk. If every set bounded in $E$ is contained in some bounded Banach disk, the space $E$ is called fast complete. It is easy to show that every quasi-complete locally convex space is fast complete.

It is well known [2, IV.5.3], that if a locally convex space $E$ is quasi-complete then $\sigma\left(E^{\prime}, E\right)$ bounded subsets of $E^{\prime}$ are $\beta\left(E^{\prime}, E\right)$-bounded. This result can be slightly generalized as follows. Assume $E$ to be fast complete and take a bounded set $A \subset E$. Then $A$ is contained in some bounded Banach disk $B$. For any $\sigma\left(E^{\prime}, E\right)-$ bounded set $H \subset E^{\prime}$, the set $\{f(x) ; f \in H\}$ is bounded for any $x$ from the Banach space $E_{B}$. Since $A$ is bounded in $E_{B}$, the set $\{f(x) ; f \in H, x \in A\}$ is bounded by the Uniform Boundedness Principle and $H$ is $\beta\left(E^{\prime}, E\right)$-bounded.

THEOREM 1. Let $E$ be a locally convex space. Then all $\sigma\left(E^{\prime}, E\right)$-bounded sets are $\beta\left(E^{\prime}, E\right)$-bounded iff $E$ is fast complete.

Proof. One implication has already been proved. Assume that $E$ is not fast complete. Then there exists a bounded and closed disk $B \subset E$ such that $E_{B}$ is not complete. Let $\left\{x_{n}\right\}$ be a Cauchy sequence in $E_{B}$ which does not converge in $E_{B}$. Since the set $A_{0}=\left\{x_{n} ; n \in N\right\}$ is bounded in $E_{B}$ and $B$ is a 0 -neighborhood, we may assume $x_{n} \in B, n \in N$.

From [3, Lemma 6-1-11], follows

Claim 1. $\left\{x_{n}\right\}$ does not converge in $E$.

Received by the editors March 18, 1986 and, in revised form, August 1, 1986.

1980 Mathematics Subject Classification (1985 Revision). Primary 46A05; Secondary 46A20.

Key words and phrases. Quasi-complete space, fast complete space, $\sigma\left(E^{\prime}, E\right)$-bounded set, $\beta\left(E^{\prime}, E\right)$ bounded set. 
The Cauchy sequence $\left\{x_{n}\right\}$ is bounded and does not have any limit. Hence it cannot be contained in a finite-dimensional space. By taking a subsequence we may assume that the set $A_{0}$ is linearly independent. Take $H_{0} \subset E$ such that $A_{0} \cup H_{0}$ is a Hamel basis for $E$ and denote by $F$ the linear hull of $H_{0}$.

There exists a balanced convex 0-neighborhood $U$ in $E$ such that $x_{n} \notin U$ for all $n \in N$. Choose and keep for the rest of the proof a balanced convex 0 -neighborhood $V$ in $E$ such that $V+V \subset U$. Since $V \cap E_{B}$ is a 0-neighborhood in $E_{B}$, there exists $n_{0} \in N$ such that $x_{m}-x_{n} \in V$ for $m, n>n_{0}$. If we discard the first $n_{0}$ terms of $\left\{x_{n}\right\}$ and renumber the sequence, we may assume $x_{n} \in x_{1}+V, n \in N$.

Claim 2. Let $A$ be the convex hull of $A_{0}$. Then $A \cap V=\varnothing$.

Assume $x \in A \cap V$. Then there are $\lambda_{k} \geqslant 0, \Sigma \lambda_{k}=1$ such that $x=\sum \lambda_{k} x_{k}=x_{1}$ $+\sum \lambda_{k}\left(x_{k}-x_{1}\right) \in x_{1}+V$ and $x_{1} \in x-v \subset V+V \subset U$, a contradiction.

Claim 3. Let $W$ be a convex hull of $V \cup F$. Then $A \cap W=\varnothing$.

Assume $x \in A \cap W$. We may write $x=\lambda y+(1-\lambda) z$, where $\lambda \in[0,1], y \in V$, $z \in F$. Further, $y=\sum \lambda_{k} x_{k}+\sum \mu_{\alpha} h_{\alpha}$ and $x-\lambda \sum \lambda_{k} x_{k}=\lambda \sum \mu_{\alpha} h_{\alpha}+(1-\lambda) z \in$ $E_{A} \cap F_{1}$, a contradiction.

The set $W$ is a balanced convex 0-neighborhood in $E$. Let $p$ be its Minkowski functional. For any $n \in N$ denote by $f_{n}$ the linear function on $E$ such that $f_{n}(x)=0$ for $x \in H_{0} \cup\left(A_{0} \backslash x_{n}\right)$ and $f_{n}\left(x_{n}\right)=1$. By Claim 2, for any $x \in A$ we have $p(x) \geqslant 1$ and $\left|f_{n}(x)\right|=\left|f_{n}\left(\sum \lambda_{k} x_{k}\right)\right|=\left|\lambda_{n}\right| \leqslant 1 \leqslant p(x)$. Hence also $\left|f_{n}(x)\right| \leqslant$ $p(x)$ for $x \in E_{A}$. For $x \in F$, we have $p(x)=0$ and $f_{n}(x)=0$. Finally, if $x \in E$, then $x=y+z$, where $y \in E_{A}, z \in F$, and

$$
p(x)=p(y+z) \leqslant p(y)+p(z)=p(y)=p(x-z) \leqslant p(x)+p(z)=p(x) .
$$

Therefore $\left|f_{n}(x)\right|=\left|f_{n}(y)\right| \leqslant p(y)=p(x)$ and $f_{n}$ is a continuous linear functional on $E$.

Put $S=\left\{n f_{n} ; n \in N\right\}$ and choose $x \in E$. We can write $x=y+\sum_{k=1}^{m} \zeta_{k} x_{k}$, where $y \in F$. Since for any $n>m, f_{n}(x)=0$ and $\sup \left\{\left|n f_{n}(x)\right| ; n \in N\right\}=$ $\sup \left\{\left|n \zeta_{n}\right| ; n=1,2, \ldots, m\right\}<+\infty$, the set $S$ is $\sigma\left(E^{\prime}, E\right)$-bounded.

On the other hand, the set $A_{0}$, bounded in $E_{B}$, is also bounded in $E$ and we have

$$
\sup \left\{\left|n f_{n}(x)\right| ; n \in N, x \in A_{0}\right\} \geqslant \sup \left\{\left|n f_{n}\left(x_{n}\right)\right| ; n \in N\right\}=+\infty,
$$

i.e. $S$ is not $\beta\left(E^{\prime}, E\right)$-bounded.

THEOREM 2. Let $E$ be a locally convex space. Then all $\sigma\left(E, E^{\prime}\right)$-bounded sets are $\beta\left(E, E^{\prime}\right)$-bounded iff $E$ is fast complete.

Proof. The $\sigma\left(E, E^{\prime}\right)$-bounded sets are $\beta\left(E, E^{\prime}\right)$-bounded iff the $\sigma\left(E^{\prime}, E\right)$ bounded sets are $\beta\left(E^{\prime}, E\right)$-bounded [3, 10.5.4]. Hence Theorem 2 follows from Theorem 1.

\section{REFERENCES}

1. Marc de Wilde, Closed graph theorems and webbed spaces, Pitman, London, 1978.

2. Helmut H. Schaefer, Topological vector spaces, 3rd ed., Springer-Verlag, Berlin and New York, 1971.

3. Albert Wilansky, Modern methods in topological vector spaces, McGraw-Hill, New York, 1978.

Department of Mathematics, Washington State University, Pullman, Washington 99164 